Annals of Plant Sciences

\title{
On the occurrence of two little known Fimbristylis (Cyperaceae) from Kerala, India.
}

\section{Rijuraj M.P.1*, Viji A.R. ${ }^{2}$, Shaju T.1, Rajendraprasad M.1, A.G. Pandurangan ${ }^{1}$}

${ }^{1}$ Plant Systematics and Evolutionary Science Division, Jawaharlal Nehru Tropical Botanic Garden and Research Institute, Karimancode P.O, Palode, Thiruvananthapuram - 695 562, Kerala, India.

${ }^{2}$ Department of Botany, University College, Palayam, Thiruvananthapuram, Kerala, India.

Received: 11/9/2017; Accepted: 11/19/2017

Abstract: Two little known Fimbristylis species have been collected from lateritic plains of Kerala. A brief description, notes and detailed illustrations are provided for easy identification.

Keywords: Cyperaceae, Fimbristylis, endemic, Kerala

\section{Introduction}

The genus Fimbristylis Vahl has about 300 species and distributed widespread in pantropical and warm temperate regions of the world with the highest concentration of species in tropical Asia (Kern 1974, Goverts et al., 2007). In India, the genus is represented by 115 species of which 59 are reported from Kerala (Prasad \& Singh 2002; Nayar et al., 2014; Viji et al., 2016).

During the intensive explorations in the lateritic region of northern Kerala, two interesting Fimbristylis species were collected by the authors, which upon a critical study had been identified as $F$. stigmatotecta Govind. and $F$. tristachya $\mathrm{R}$. Br. Fimbristylis stigmatotecta Govind. was described by Govindarajalu in 1996 based on the collections from Lockhart gap, Devikulam, Idukki district, Kerala (Type: CAL 15126) and there after no collection of this species has been reported so far from India. Hence, the present collection is not only confirmed its existence in Kerala but also forms a recollection of this species after a lapse of two decades. Fimbristylis tristachya R. Br., a perennial sedge species is widely distributed in tropical Asia to North West Pacific and the known distribution of this species in Kerala is from Chinnar Wildlife Sanctuary in Idukki district (Sasidharan, 2004). The present report of this highland species from the lateritic regions is confirmed the extended distribution to the low land regions. Detailed descriptions with illustrations, colour photographs and relevant notes are provided to facilitate easy identification.
Taxonomic Treatment:

Fimbristylis stigmatotecta Govind., Rheedea. 6(2): 61. 1996. (Fig. 1 \& 2)

Type: India, Kerala, Idukki district, Lockhart gap, Devikulam, Govindrajalu 15126 (Holotype: CAL; Isotypes: MH, BSI, BLAT, DD)

Annual herbs, $20-35 \mathrm{~cm}$ tall. Roots fibrous. Culms solitary or tufted, sulcate, compressed, smooth, 18 $30 \mathrm{~cm}$ high, $1-1.5 \mathrm{~mm}$ thick, swollen at base. Leaves basal, lower ones reduced to sheaths, upper bladed; sheaths laminiferous, glabrous, many nerved, mouth oblique; blades linear, $7-25 \mathrm{~cm}$ long, $0.5-1$ $\mathrm{mm}$ wide, erect or slightly curved, flat or with thickened margins, acuminate, 3-5 nerved. Inflorescence simple, loose, $1-5$ spikelets, $1-3 \times 1-$ $2 \mathrm{~cm}$; involucral bracts much shorter than inflorescence, erect or suberect, lanceolate, smooth, glabrous, 5 - $10 \mathrm{~mm}$ long; primary rays up to 5, slender, 0.5 - $3 \mathrm{~cm}$ long, obliquely patent, compressed, glabrous, smooth. Spikelets solitary, elliptic ovoid or ellipsoid, angular, acute, 8-12 flowered, $8-10 \times 2-4 \mathrm{~mm}$, ferrugineous castaneous brown; rachilla narrowly winged; glumes spiral, chartaceous, obliquely erect, ovate-oblong, 3 -3.8 x $1-2 \mathrm{~mm}$, apex obtuse, mucronate, scarcely keeled, many nerved, nerves excurrent into mucro, fulvous-ferrugineous, hyaline margins. Stamens 3; anthers linear, $1-1.5 \mathrm{~mm}$ long; filaments short. Ovary obovoid, 0.3 - $0.5 \mathrm{~mm}$ long; style flat, broad, ca. 2 $\mathrm{mm}$ long, slightly dilated at base; stigmas 2, 0.8 - 1 $\mathrm{mm}$ long. Nutlets globosely obovoid, rounded at apex, smooth, stipitate, $1-1.5 \times 0.5-1 \mathrm{~mm}$, yellowish brown or blackish brown, epidermal cells in upper half distinct, hexagonal, arranged in 10-15 regular rows on each face.

\footnotetext{
${ }^{*}$ Corresponding Author:

Mr. M.P.Rijuraj.,

Plant Systematics and Evolutionary Science Division,

Jawaharlal Nehru Tropical Botanic Garden and Research Institute,

Karimancode P.O, Palode, Thiruvananthapuram - 695 562, Kerala, India.

E-mail: rijurajmp@gmail.com
} 

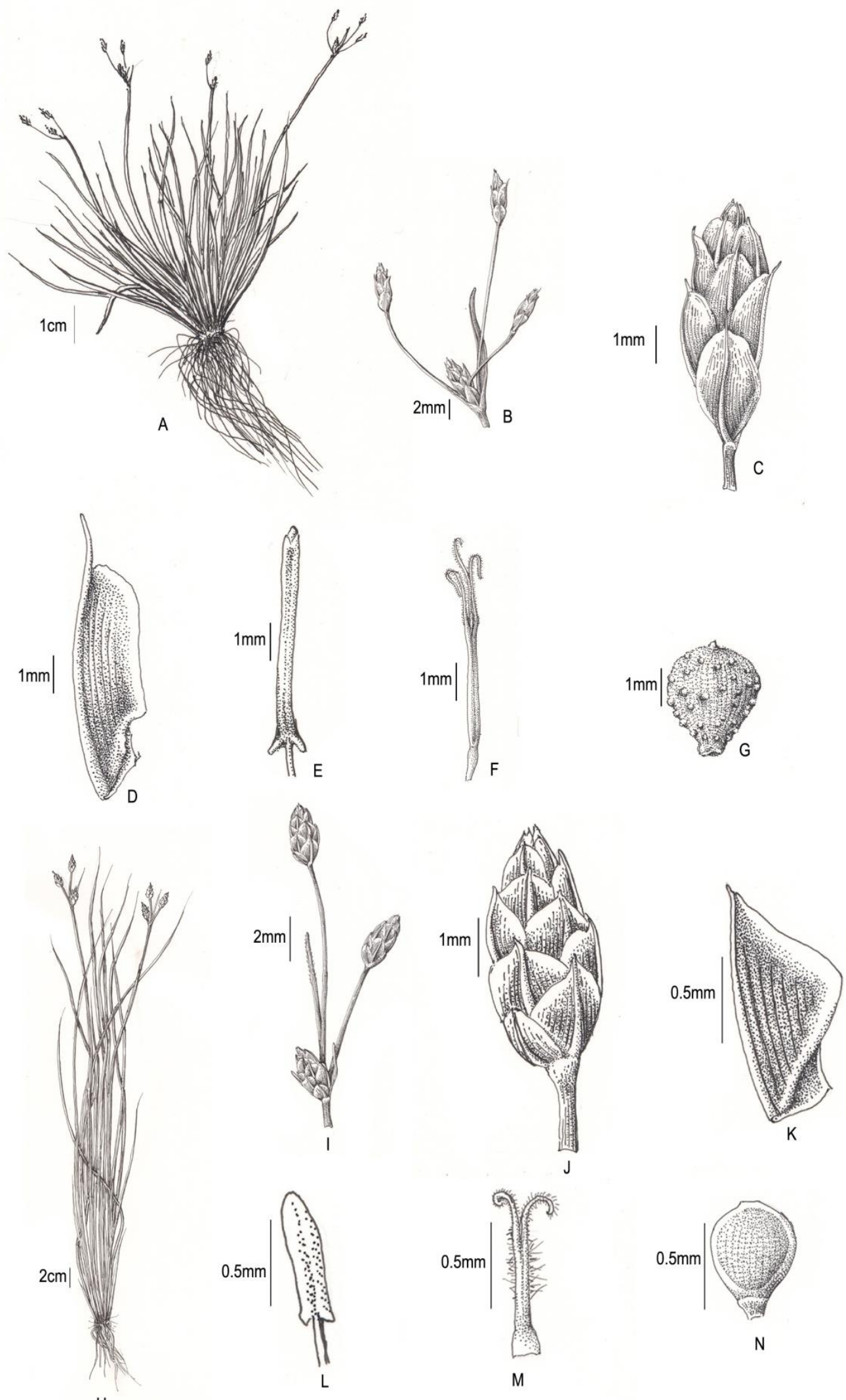

$\mathrm{H}$
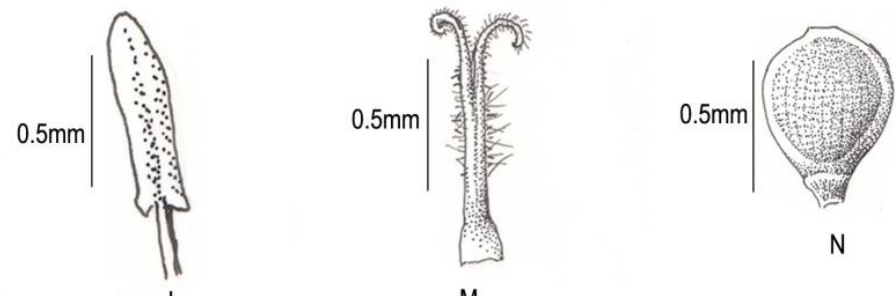

Figure 1. Fimbristylis stigmatotecta Govind. A. Habit, B. Inflorescence, C. Spikelet, D. Glume, E. Anther, F. Style \& Stigma, G. Nutlet.

Fimbristylis tristachya R. Br., H. Habit, I. Inflorescence, J. Spikelet, K. Glume, L. Anther, M. Style \& Stigma, N. Nutlet. 

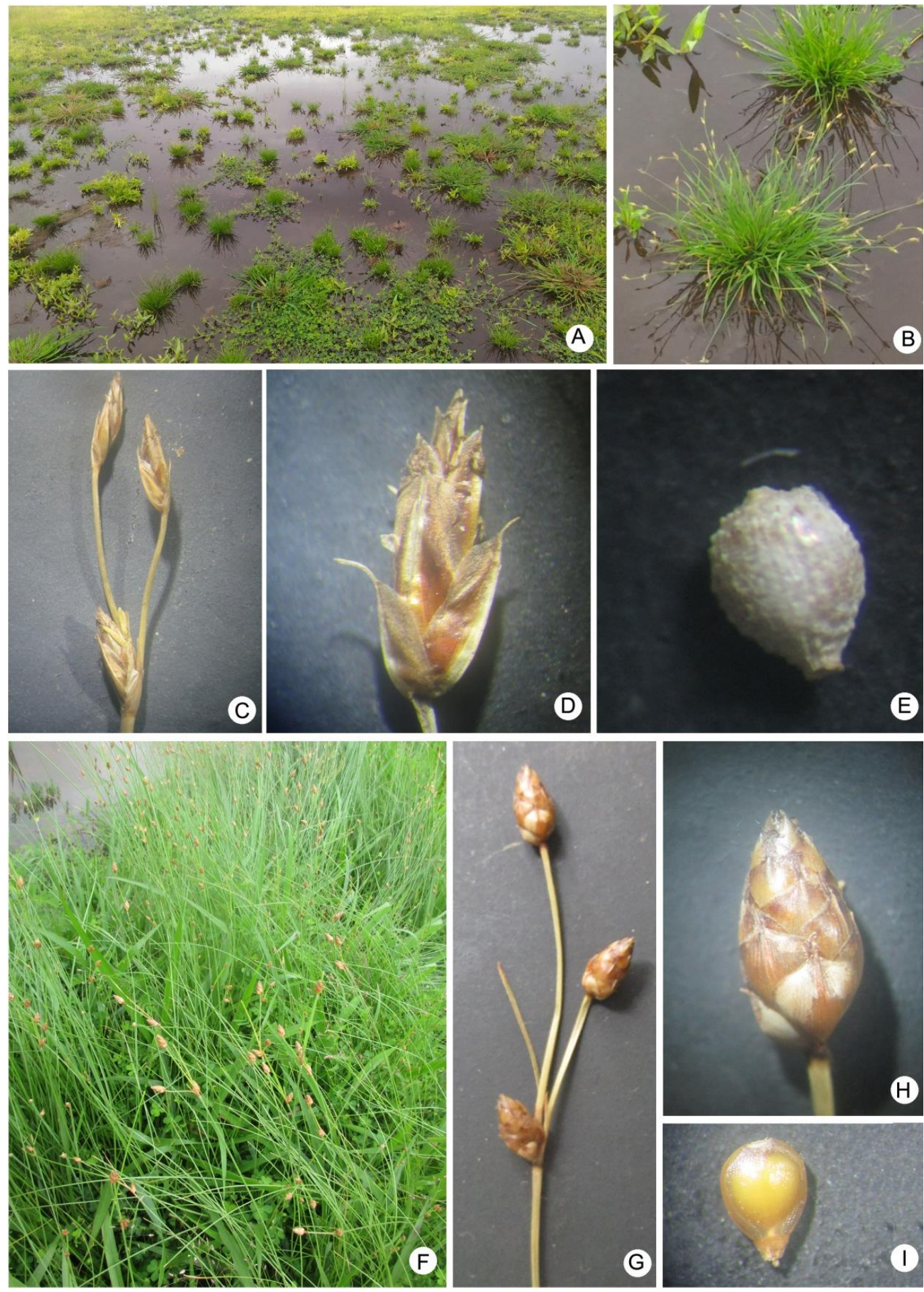

Figure 2. Fimbristylis stigmatotecta Govind. A. Habitat, B.Habit, C. Inflorescence, D. Spikelet, E. Nutlet.

Fimbristylis tristachya R. Br., F. Habitat, G. Inflorescence, H. Spikelet,I. Nutlet.

Phenology: July to December

Habitat \& Ecology:

It is naturally growing in the waterlogged seasonal Dopatrium junceum (Roxb.) Buch.-Ham. ex Benth., pools of Madayipara in Kannur District, Kerala. Isachne bhatii Biju et al., Murdannia crocea (Griff.) The associated species in the community are Faden ssp. ochracea (Dalz.) Faden, etc.

Geissaspis tenella Benth. var. tenella Hook. f., 
Specimen examined: India, Kerala, Kannur district, Madayipara, \pm 42 m, 20 July 2017, Shaju, Rijuraj \& Rajendraprasad, 90946 (TBGT)

Fimbristylis tristachya R. Br., Prodr. Fl. Nov. Holl. 227. 1810. (Fig. 1 \& 2)

Type: Australia, Nov 1802 R. Brown. 5941 (000990944, image, BM!)

Perennial herbs, $50-100 \mathrm{~cm}$ tall. Rhizome shortly creeping, covered with ovate, striate, brownish sheaths. Culms tufted, sulcate-angular, compressed, smooth or scabrid upwards, $40-95 \mathrm{~cm}$ high, $1-$ $1.5 \mathrm{~mm}$ thick. Leaves basal, lower ones reduced to sheaths, upper bladed; sheaths coriaceous, brownish; ligule a dense fringe of short hairs; blades linear, 10 $25 \mathrm{~cm}$ long, $0.5-1.5 \mathrm{~mm}$ wide, greyish green or glaucous, erect or slightly curved, flat or with inrolled margins, acuminate, scabrid on the margins in the upper part. Inflorescence simple or subcompound, loose, $1-7$ spikelets, $1-5$ x $2-4$ $\mathrm{cm}$; involucral bracts much shorter than inflorescence, erect or suberect, scarious- margined at dilated base; primary rays up to 5, slender, 0.5 - 3 $\mathrm{cm}$ long, obliquely patent, compressed, glabrous, smooth. Spikelets solitary, ovate or ovate-lanceolate, terete, acute, densely many flowered, 5 - 25 x 4 - 6 $\mathrm{mm}$, brown; rachilla narrowly winged; glumes spiral, chartaceous, obliquely erect, broadly ovate, 4 - 6 x 2 - $4 \mathrm{~mm}$, apex obtuse, mucronate, scarcely keeled, many nerved, fulvous-ferrugineous, hyaline margins. Stamens 3; anthers linear, $1.5-2.5 \mathrm{~mm}$ long; filaments short. Ovary obovoid, 0.3 - $0.5 \mathrm{~mm}$ long; style flat, broad, $2 \mathrm{~mm}$ long, slightly dilated at base; stigmas 2 , $0.8-1 \mathrm{~mm}$ long. Nutlets biconvex, obovoid, $1-1.5$ x $0.5-1 \mathrm{~mm}$, smooth, stramineous, obscurely reticulate by the isodiametric epidermal cells.

Phenology: July to November

Habitat \& Ecology:

It is naturally growing in small populations in marshy areas on the lateritic rock surface of Peringome village in Kannur District, Kerala. The associated species in the community are Geissaspis tenella Benth. var. tenella Hook. f., Eragrostis sp., Dimeria sp., Rotala malampuzhensis R.V. Nair ex Cook, etc.
Specimen examined: India, Kerala, Kannur district, Peringome, \pm 55 m, 18 July 2017, Rijuraj, Viji \& Shaju, 90917 (TBGT)

\section{Acknowledgements}

The authors are grateful to the Director, Jawaharlal Nehru Tropical Botanic Garden and Research Institute (JNTBGRI), Palode, Thiruvananthapuram extended support are help in research activities. And also are thanking to the Kerala State Council for Science Technology and Environment, Kerala, for providing financial support and first named author is grateful to Kerala University, Thiruvananthapuram.

\section{References}

1. Govaerts, R., Simpson, D.A., Goetghebeur, P., Wilson, K.L., Egorova, T. and J. Bruhl. 2007. World checklist of Cyperaceae. The Board of Trustees of the Royal Botanic Gardens, Kew.

2. Kern, J. H. 1974. Cyperaceae. In: C. J. G. van Steenis, ed. Fl. Males. ser. 1.7 (3):435-753.

3. Prasad V. P. and N. P. Singh, 2002. Sedges of Karnataka (India). Scientific Publishers, Jodhpur.

4. Sasidharan, N. 2004. Biodiversity Documentation for Kerala. Part 6. Flowering Plants of Kerala. Forest Research Institute, Peechi.

5. Nayar, T. S., Rasiya Beegam, A. \& Sibi, M. (2014). Flowering plants of The Western Ghats, India. Jawaharlal NehruTropical Botanic Garden and Research Institute, Kerala.

6. Viji, A. R., Pandurangan, A. G. and Deepu Sivadas 2016. Fimbristylis tuckeri (Cyperaceae), a new sedge species from the Western Ghats India. Kew Bulletin. 71: 38.

\section{Cite this article as:}

M. P. Rijuraj, A. R. Viji, T. Shaju, M. Rajendraprasad \& A. G. Pandurangan. On the occurrence of two little known Fimbristylis (Cyperaceae) from Kerala, India. Annals of Plant Sciences 6.12 (2017) pp. 1862-1865.

doi: http://dx.doi.org/10.21746/aps.2017.6.12.10

Source of support: Jawaharlal Nehru Tropical Botanic Garden and Research Institute (JNTBGRI), Palode, Thiruvananthapuram

Conflict of interest: Nil 